\title{
Die venerischen Krankheiten bei den alten Aegyptern.
}

Eine historische Skizze von J. K. Proksch in Wien.

Ein neues Verbindungsglied der immer noch mehrfach durchbrochenen Kette von den Kenntnissen über die Geschichte der venerischen Krankheiten seit ihrem Ursprunge bei den zeitlichsten Völkern des Alterthums bis zur richtigen Beurtheilung der Syphilis im Beginne der Nenzeit scheint gefunden zu sein. Zwar sind die Räume, welche hier durchwandelt werden müssen, trotz wancherlei in den letzten Jahrzehnten aufgefuudenem Beleuchtungsmaterial noch nicht genügend erhellt, um rasch und sicheren Schrittes zum Ziele gelangeu zu können, aber es sind doch nicht mehr die subjectiv gefärbten, mitunter flüchtig hingezeichneten Bilder der alten Griechen und Römer, sondern es sind wirkliche, greifbare Erzeugnisse des alten Aegypten, welche nun der historischen Untersuchung zugängig gemacht wurden.

Eine auffallende, zu strengster Objectivität auffordernde Erscheinung ist es jedoch, dass ungeachtet der Schätze, welche unser Jahrhundert über die Geschichte der alten Aegypter zu T'age gefördert hat, die (ielehrten der Gegenwart iiber den Werth und die Bedeutung der ägyptischen Heilkunde eben so wenig einig sind, wie die alten Griechen. Schon in den Homerischen (iedichten ${ }^{1}$ ) werden die ägyptischen Aerzte als die Nachkommen des Götterarztes Paieon, welche alle Sterblichen an Erfahrungen überragen, besungen; und auch spätere Reisende, (Geschichtsforscher und Philosophen wie II e rod ot,") Diod or, ${ }^{3}$ ) Clemens von Alexandria ${ }^{4}$ u. A. berichten rühmend sowohl iuber die Grossartigkeit der Bautenkmale als auch über die Weisheit der agyptischen Priesterärzte; aber schon Gale- 
nus ${ }^{5}$ ) erklärte die hermetischen Bücher der ägyptischen Heilkunde, die er allerdings für unecht und unterschoben hielt, für albernes Zeug und eitel Thorheit. In ähnlicher Weise stehen sich auch heute wieder zwei Parteien einander gegenüber. Während die Aegyptologen von Fach, an ihrer Spitze Ebers: für unzweifelhaft halten, „dass ein beträchtlicher Theil der medicinischen Schriften der Griechen aus Aegypten stammt"; sonach die Anfänge wissenschaftlicher Heilkunde diesem, und nicht wie bisher jenen zugeschrieben werden müssten, erklären anderseits die medicinischen Historiker von Fach, voran August Hirsch, ${ }^{7}$ ) die Medicin der alten Aegypter für „roh-empirisches, mit theurgisch-mystischen Träumereien durchsetztes Handwerk". Gleichsam vermittelnd trat jüngst Heinrich J o a chim, ${ }^{\mathbf{s}}$ ) Arzt und Aegyptolog, auf: er unterlässt es ganz die Verdienste der Griechen zu schmälern und die der Aegypter zu erheben, rübmt diesen jedoch wiederholt nach, dass sie „vorzügliche Beobachter waren".

Ohne näher auf den Grund dieser Meinungsverschiedenheiten einzugehen, mag hier nur so viel zur Charakterisirung der alten Aegypter hervorgehoben werden, dass sie, wie die bisher aufgefundenen: Papyri unwiderleglich nachweisen, von der Anatomie, und allem was herum und daran hängt, nicht die geringfügigsten Anfänge wissenschaftlicher Kenntnisse besassen und in therapeutischer Beziehung bei ihnen nur, wie dies August $\mathrm{Hirsch}$ sehr kräftig und ein wenig an Wi p p chen gelehnt ausdrückt, "die Dreckapotheke in voller Blüthe stand". Freilich fehlt noch Vieles zur richtigen Beurtheilung der medicinischen Anschauungen dieses alten Culturvolkes, denn nach den glaubwürdigen Berichten von $\mathrm{Clemens}$ Alexandrinus, Jamblichus ${ }^{9}$ ) u. A. waren sechs hermetische Bücher über

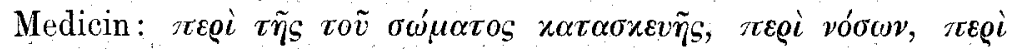

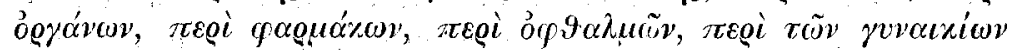
(vów(uv) vorhanden, von denen vorläufig im Papyrus Ebers nach der Meinung des gelehrten Entdeckers erst das vierte vorliegt. Dasselbe ist höchst wahrscheinlich bereits in der Mitte des sechszehnten Jahrhunderts vor unserer Zeitrechnung niedergeschrieben, vielleicht aber noch früher, wenigstens in einzelnen Abschnitten, verfasst worden. 
Die vor diesem aufgefundenen viel kleineren medicinischen Papyri mögen wohl nur Auszüge aus den hermetischen Büchern enthalten. Da diese Papyri theils; wie die zwei von $\mathrm{Brugsch}$ im Berliner Museum, noch nicht übersetzt, theils, wie der Papyrus des British Museum, heute noch nicht einmal edirt, oder, wie die Papyri in Leyden, Turin, Paris und Boulay̆, nur magisch-medicinischen Inhalts sind, so kommt für den Nichtkenner der hieroglyphischen und hieratischen Schriftarten bloss der allerdings weitaus umfangreichste, fehlerfreieste, schönste und vollständige Papyrus Ebers in Betracht, welcher unlängst von Heinrich Joachim in deutscher Uebersetzung herausgegeben wurde. Edirt wurde derselbe in elegantester Ausstattung bereits in Jahre 1875. Der Papyrus Ebers ist, wie schon aus rem Titel, welchen der Entdecker dem Werke gegeben hat und aus der Aulschrift des Papyrus selbst erhellt, eine Armeimittellehre oder eigentlich eine Receptensammlung. Die wenigen anatomischen, pathologischen und chirurgischen Bemerkungen, welche hie und da eingeflochten erscheinen, berechtigen wohl kaum dazu die Schrift mit H. Joa chim für "eine Art medicinisches Compendium" zu halten. Doch überlassen wir dies und überhaupt alle äusserlichen Subtilitäten den Aegyptologen und wenden uns nun zu dem Inhalt des Werkes.

Die darin vorkommenden Receptformeln sind nicht nach der naturhistorischen Abstammung irgend eines orler mehrerer Hauptmittel, oder, wie dies im Alterthum geliäuchlich war, nach ihrer Verwendung bei len verschiedenen Krankheiten a capite ad calcem geordnet, sondern beginnen mit den Arznejformeln gegen "die Krankheiten im Leib", denen ohne irgend welche erfindbare systematische Aneinanderreihung einige constitutionelle Erkrankungen, dann die Krankheiten des Mundes, der Haut, des Anus, eine "töltliche Krankheit" wieder "im Leib", hierauf liumpf, Magen, Herz, Kopf u. s. w. unter- und durcheinander folgen. Da wir also im Papyrus kbers keine Pathologie und auch kein medicinisches Compendium ror uns babon, so können wir über die einzelnen Krankheiten keine näheren Aufschlïsse erwarten, und mïssen uns mit der bisher leider noch nicht vollständig aufgeklärten Nomenklatur abfinclen, welche den einzelnen Abschnitten und Recepten als Aufschrift 
dient. Es dürfte sich kaum ein zweites medicinisches Werk des Alterthums, namentlich kein pharmakologisches, finden, in welchem den Erkrankungen des Greschlechtsapparates und seiner Umgebung ein verhältnissmässig so breiter Raum angewiesen ist, als im Papyrus Fbers; wohl ist in demselben mit keinem Worte gesagt, dass diese Erkrankungen durch Ansteckungen aus dem geschlechtlichen Verkehr entstanden sind, doch fehlen ja solche Angaben regelmässig in den Receptensammlungen aller Zeiten, aber dennoch lassen sich die jeweilig namhaft gemachten Erkrankungen auf Grund der historischen Pathologie deuten. Am reichlichsten ist die Zahl der Localaffecte an den weiblichen Geschlechtstheilen; die hier interessirenden Stellen sind folgende:

Taf. 95. „Ein anderes (Recept) den Flour (albus) nicht ïber die Jungfrau kommen zu lassen." ${ }^{10}$ )

"Mittel Geschwülste in der Vulva (Uterus?) zu vertreiben: Getrocknete zet-Blätter in Hefe von starkem Bier auf ihre Weiche und ihren Leib bringen."

„Ein anderes (Mittel) gegen Fressen in der Vulva, das bennut ${ }^{11}$ )-Blasen in ihrer Scheide hervorbringt: Frische Datteln 1, hekennu ${ }^{12}$ )-Körner 1, Stein von der Flussmündung, zerreiben in Wasser, feucht stehen lassen und in ihre Vulva spritzen."

„Ein anderes: Frische Datteln 1, Schweingalle 1, genti ${ }^{13}$ )Körner 1, Wasser, feucht stehen lassen und in ihre Vulva spritzen."

„Ein anderes gegen Entstehen von Krankheiten in ihrer Schamlippe: Knoblauch 1, Grüne Bleierde (?) 1, nehedet ${ }^{14}$ )Körner 1, Weihrauch 1, Harz von Acanthus 1, Kuhhorn 1, hunta ${ }^{15}$ )-Körner 1, Wasser 1, in Eins machen und in die Vulva spritzen."

Taf. 96. "Ein anderes gegen Stiche in der Vulva und gegen runde Pusteln, die in ihrer Scheide entstehen: zeper-urKörner 1 zerreiben in Weihrauch-Wasser 1, genti-Körner 1, in ihre Vulva spritzen."

„Ein anderes: nāh-Korn 1/s, frische Datteln 1/s, Harz von Acanthus $1 / 8$, genti.Körner 1/32, Wasser 1/3, Eselsmilch, feucht stehen lassen und in ihre Vulvaa spritzen."

"Ein anderes die Vulva (Uterus?) zu kühlen und Entzün- 
dung darin zu vertreiben: Dumpalmenfrucht zermahlen, Cyperus zermahlen in Oel und in die Vulva spritzen; es ist ein zusammenziehendes Mittel für die Vulva (den Uterus)."

"Ein anderes: Sesamum in Honig zermahlen und in ihre Vulva spritzen; es ist ein zusammenziehendes Mittel."

„Ein anderes: Weihrauch und Crocus in Kuhmilch zermahlen und zerreiben, durch ein Tuch durchseihen und in ihre Vulva spritzen; es ist ein zusammenziehendes Mittel."

"Ein anderes die Vulva (den Uterus) zusammen zu ziehen : zeper-ur-Körner 1, Honig 1, Zwiebelwasser 1, Milch 1, durchseihen und in ihre Vulva spritzen lassen."

„Ein anderes: Wasser von mes9 $9 \bar{a}$-Getränk in ihre Vulva spritzen."

"Ein anderes: Saft von der neša ${ }^{16}$ )-Pflanze in ihre Vulva spritzen." spritzen."

„Ein anderes: Saft von quereqtu-Körnern in ihre Vulva

"Ein anderes: Pfefferminzwasser in ihre Vulva spritzen."

"Wenn Du ein Weib untersuchst, es gebt etwas von ihr ab wie Wasser, dessen Bodensatz wie warmes Blut ist, so sag Du ihr: es ist die axāt ${ }^{17}$ )-Krankheit in ihrer Vulva. Mach Du für sie Jaspis, der Wasser anzieht; reiben in Honig und Collyrium, Charpie von Leinwand damit bestreichen und 4 Tage in ihre Vulva thun."

'T'af. 97. „Ein anderes Mittel gegen Intzündungsschmerzen un die Vulva (den Uterus): Kuhgalle 1, genti-Körner 1, Oel 1 in Fins machen und in ihre Vulva spritzen."

Andere in differential-diagnostischer Beziehung interessirende Stellen können wegbleiben, da die vorgeführten wohl genügenden Aufschluss über verschiedene Behaftungen geben, von denen die venerischen gewiss nicht ausgeschlossen werden können. Minder zahlreich sind Erkrankungen an den männlichen Genitalien erwähnt.

Taf. 49. „Ein anderes (Recept) Verstopfung (oder Verhärtung) auf (irund von Entzündung im Uringang bei einer Person zu vertreiben, die an Verstopfung des Irins leidet: Seesal\% 1/64, mahui 18)-Samen 1/a, Baumöl 1, Honig 1, süsses Bier 1, in den Anus $/$ u spritzen." 
„Audere Mittel gemacht für den, der krank ist mit uxedu in seinem Uringang: Kuhleber 1, anest-Pflanze 1, thun in pătBrod und von der Person zu essen."

"Ein anderes: dešer ${ }^{19}$ )-Samen 1/8, das Inwendige von Zwiebeln $1 / 32$, Weizenmehl $1 / 4$, Gänseschmalz $1 / 8$, Honig $1 / 8, \mathrm{~Pa}-$ pyruspflanze $1 / 8$, Wasser $1 / 2$, kochen, durchseihen und 4 Tage lang einnehmen."

Taf. 50. "Andere Mittel zu viel Urinausleerung zu vertreiben: Weizenkorn $1 / 8$, Sebesten $1 / 8$, Grüne Bleierde (?) $1 / 32$, Wasser $1 / 2$, feucht stehen lassen, durchseihen und 4 Tage einnehmen."

Hier dürfte es vielleicht heissen, „zu ofte" statt "zu viel“" Urinentleerung, denn dann passt besser das unmittelbar Folgende:

"Ein anderes das zu schnelle Urinlassen zu vertreiben: Wachholderbeeren 1, Cyperus 1, Bier 1, kochen, durchseihen und 4 Tage lang einnehmen."

"Mittel den Urin herauszutreiben: Crocus des Berges $1 / 4$, Crocus des Delta 1/8, abu-Pflanze von Oberägypten 1/16, Beere vom uān-Baum 1/16, frische Grütze $1 / s$, abu-Pflanze von Unterägypten $1 / 16$, Leinsamen (?) $1 / 16$, uam-Samen $1 / 16$, duat-Pflanze $1 / 16$, Wasser $1 / 16$, feucht stehen lassen, durchseihen, 4 Tage lang einnehmen."

H. Joachim vermuthet unter letzterem eine Strictur. Erwägt man, dass ausser diesen noch Harnbeschwerden anderer Art, namentlich auch bei Kindern erwähnt werden, so erhält man aus dem Zusammenhang der vorgeführten Stellen den Eindruck, als ob wir es hier mit dem Tripper und seinen Erscheinungen bezüglich der Urinexcretion zu thun hätten; befremdend ist nur, dass der Ausfluss mit keinem Worte erwähnt ist; nur Taf. 71 finden sich drei Recepte gegen "Ausflüsse aller Art", ihre Heilung geschieht jedoch durch Pflaster. Aber dennoch ist nicht anzunehmen, dass der "Eiterfluss“ des Gliedes; welcher im Leviticus beschrieben und auch an anderen Stellen der Bibel genannt wird, den alten Aegyptern unbekannt geblieben wäre; es ist vielmehr wahrscheinlich, dass der ,Eiterfluss", als bei der "Entzündung im Uringang": allgemein bekannt, eben deshalb nicht erwähnt wird, umsomehr als eine direkte topische Behandlung desselben nicht im Gebrauch war. 
Dafür spricht doch auch die vorerwähnte Prophylaxis gegen Fluor albus, und "gegen Entstehen von Krankheiten in ihrer Schamlippe".

Die ulcerösen Affectionen an den männlichen Genitalien werden ebenfalls weniger eingehend erörtert, als an den weiblichen. Heinrich L. Emil Lür íng ${ }^{20}$ ) übersetzt eine Stelle des Papyrus Ebers folgend:

Taf. 86. „Anfang der Recepte zur Vertreibung des šepen ${ }^{21}$ )Geschwüres, welches das Fleisch eines Mannes oder einer Frau umwickelt (a. h. überzieht): Salz rom Norden, Weibrauch, frische Milch, klystiren in den After, es wird anch gemacht, ohne Weihrauch hinzuzuthum."

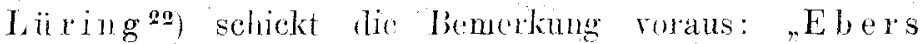
spricht von šepen-Entzündung. Wodurch die Entzündung in dem Worte bereichuet jst, sehe ich nicht, mir scheint es vielmehr ein schmerzhaftes Gesehwiir zu sein, das am Coterleibe dros Menschen mit Trin und After in Virbindung gebracht werden komnte." - Ineses klingt anch im pathologischen Sinne um so wahrscheinlieluer, als hier das Wort . lleisch" jechenfalls in euphemistischer Bedratung fïr Geschechtstlieil gebraucht wird und rann auch rime dem swoitnärhst folgenden Recepte im Papyrus beigefügte Bemerkung cinigermassen verständlich wird:

, Hin anderes (gegen šepen-(ieschwiir): Eusche Milch $5 / 6$, Baumöl (?) $5 / 6$, Kupferrost $1 / 16$. Collyrium $1 / 16$, Honig $1 / \mathrm{s}$, ebenso (nu klystiren in den After). lis ist beriihmt die uyedu zu vertreiber." Welche Bewandtniss die uyedu nit dem Geschwüre hat, worden wir baht schen. Ausserdem folgrn dem eben Angefülıten unmittelbar:

"Mittel ein stinkendes Geschwür im Sommer zu vertreiben: Weihrauch 1, ahn-Pfauzo 1, Wachholderberen 1, Myrohen 1, in Fins samroehn und damit salben."

"Fin anderes stinkendes Grschwiir im Körper ${ }^{23}$ ) (Fleisch, Geschlechtstheil?) eines Mannes oder (inex Kran zu rertreiben."

„Ein andores: Weihrauch, Toig, in Eins mischen, in eine Kugel machen; anf den Ort thun, wo ein Glied sich mit dem andem verbindet." (i) lebersetzung des letzen Passus erklint H. Joach im für "nirlet sicher".) 
Ganz ohne Belang dürfte es nicht sein, dass diesen Stellen "Mittel Ausschlag (Grind) im (am?) Kopfe zu vertreiben" unmittelbar folgen. Das Wort šepen wird von anderen Aegyptologen verschieden als Impetigo, Scabies, Räude und rothe Flecke übersetzt: H. Joa chim weicht dem allen aus und setzt šepen-Krankheit.

In der Uebersetzung als unsicher erklärt ist ferner folgende Stelle:

Taf. 39. „Es hat sich ein Geschwür gebildet; es ist fauler Eiter und Fluss von der Wunde. ${ }^{24}$ ) Mach ihm die Mittel, die es (das Geschwür) durch Arzneimittel öffnen. Wenn Du eine Person untersuchst, die das Leiden an ihrem ro-ab ${ }^{25}$ ) hat, ihre Glieder sind zart und ganz ermattet; Du untersuchst sie und findest keine Wunde ${ }^{26}$ ) (Geschwüre) am Leib ausgenommen an den Geschlechtsorganen ${ }^{27}$ ) wie ein Kügelchen, so sag Du zu ihr: es ist Gefährliches in Dir."

An Bubonen gemahnt das Folgende:

Taf. 51. „Der Beginn von den Arzneimitteln, Anschwellungen in (ler Leiste ${ }^{2 s}$ ) zum Fallen zu bringen: Eine Pflanze, senenutat ${ }^{29}$ ) mit Namen, die auf ihrem Bauch gleich der qadetPflanze kriecht und blüht wie die Lotuspflanze, so dass man ihre Blätter gleich einem weissen Baum findet. Man bringe sie herbei und lege sie auf die Weiche (Leiste); die Schwellung fällt sogleich. Auch wird ihr Samen in Brod auf die uzedi gethan um sie in der Weiche zum Fallen zu bringen."

Taf. 52. "Ein anderes (Drüsen-) Anschwellungen im Leib (Genitalien?) eines Mannes oder einer Frau zu vertreiben: Feigen $1 / 8$, Sebesten $1 / 8$, Weinbeeren $1 / 16$, Weihrauch $1 / 64$, Kümmel $1 / 64$, Kräuter des Feldes $1 / 8$, Honig $1 / 8$, süsses Bier $1 / 8$, durchseihen und einnehmen."

Taf. 107. "Wenn Du einen Eiter.Tumor in einem beliebigen Glied einer Person triffst und findest die Spitze davon erhöht, begrenzt und mit rundlicher Form, so sag Du dazu: Es ist ein Eiter-Tumor, der in seinem Fleisch umläuft. Ich werde die Krankheit mit dem Messer behandeln. Es ist etwas wie Milchsaft darin, darnach kommt etwas (Hässliches) heraus wie Wachs, es ist sackförmig; wenn etwas in seinem Sacke übrig (zuriick) bleibt, so rollt es herum (macht Hohlgänge?)." 
Dieses wären, wenn man von etlichen weniger Sicherem absieht, die Hauptstellen im Papyrus Ebers, welche sich auf örtliche venerische Affectionen an den Geschlechtstheilen beziehen lassen. Dieserwegen würde es sich wohl kaum verlohnt haben, weitläufige Untersuchungen auf einem bisher noch so wenig urbar gemachten Gebiete vorzunehmen, denn zuverlässliche Anhaltspunkte für die Existenz des Trippers, Schankers, der Bubonen und Feigwarzen im Alterthum finden sich in hinlänglicher Menge wohl in allen Literaturen. Was die Historiker auf diesem Gebiete seit fast vier Jahrhunderten veranlasste immer and immer wieder die alten und neuentdeckten Quellen der grauen Vorzejt zu durchmustern, waren nicht die primären venerischen Affectionen, sondern es war die Syphilis. Von dieser geben uns aber gerarle die Aegyptologen bisher nicht die mindeste Andeutung; sogar die Scliwester-Krankheit, oder wie F. A. Simon ${ }^{3 \prime \prime}$ ) will die Mutter und wiederum Tochter der Syphilis, die Lepra, ist im Papyrus Fbers nur ein einziges Mal, und cha anch nur fraglich erwälnt. ${ }^{31}$ ) Und dennoch kömnen wir aus ler bibel mit einiger Sicherheit schliessen, lass beide, sowohl Lepra als anch Syphilis den alten Aegyptern bekinnt gewesch sein müssen, demn das 13. Capitel des Leviticus kann min den Israeliten wohl nicht zumuthen.

Der Papyrus Eber's führt eine Reihe von Krankheitsnamen, welche die Aegyptologen bisher noch nicht iibersetst, sontern nur in allgemein bekannte Schriftzeichen iibertragen hallen. Findlet sich vielleicht unter diesen translatirten, unbekannten Krankheitsmamen die gesuchte Lepra und Syphilis? rersuchen wir es mit letzterer.

Fine im Papyrus libers sehr oft genannte wnd auch hier bereits dreimal in Verbindung mit den verschiedenen Affektionen an den Genitalion vorgekommene Krankheit oder

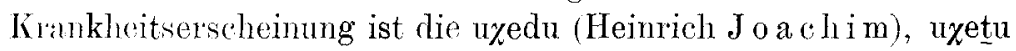
(Heinrich L. Limil Lüring), Uchet (Heinrich Haeser, ${ }^{\mathbf{3 2}}$ ) Brugsciln?). Was vermuthen also die Aegyptologen unter dieser uyedu? Die Trtheile von Brugsch und I üring ${ }^{\mathbf{3}}$ ) können wir mitsammen abhören; Letzterer sagt: „Ebers und Stern sehen in whan den Stein, die Steinkrankheit. Gegen diese Annahme spricht, dass die Krankheit nie mit dem Urine 
zusammengebracht wird; was doch bei der Besprechung derselben nicht hätte unterlassen werden können. Die etymologische Bedeutung ist: die verheerende, vielleicht die verbrennende Krankheit. Sie steht in naher Beziehung zu den uzetu und die Frklärung " "es ist eine Schwere in seinem Leibe" "s passt besser auf eine starke Indigestion, als auf Lithiasis (die bei der schlechten Verdaung sich findende Härte des Leibes scheint als ein innerliches Geschwür aufgefasst worden zu sein!). Eine ähnliche Bedeutung muss u\%etu haben, obgleich man im ersten Augenblicke an die allgemeine Uebertragung " schmerzen"." denken wird. Brugsch rergleicht mit Wahrscheinlichkeit (die uxetu) mit pinguis, crassus, obesus, crassities, pinguedo. So liegt es nahe, an die Bedeutung: „ "Geschwulst, Anschwellün"“ “ zu glauben, umsomehr als wir Blut-uyetu und offene Wunden in den uzetu verzeichnet finden; wo die Bedeutung " "Schmer" zen"." ausgeschlossen ist." - Joa chim ${ }^{34}$ ) erklärt: „wzedu ist gleich schmerzhafte Anschwellung “, zählt dann mehrere Körpertheile auf, an denen diese Erscheinung vorkommt und gelangt dann zu dem wahrhaft überraschenden Ergebniss, dass man unter uzedu "die bei der Chlorosis aegyptiaca auftretende schmerzhafte Anschwellung des Leibes zu verstehen hat." Joachim sieht überhaupt die Chlorosis aegyptiaca auf allen 108 Tafeln des Papyrus Ebers, denn die āaă-Krankheit ist nach Jo a c h i m die Chlororis aegyptiaca selbst und ausserdem ist noch die uha-Krankheit die bei der Chlorosis aegyptiaca vorkommende chronische Obstipation und der Meteorismus des Unterleibes. Da also die Aegyptologen keinesfalls wissen was uzedu ist und in ihren Vermuthungen miteinander nicht übereinstimmen, wollen wir das von dieser Krankheit im Papyrus Ebers Gesagte, selbst und möglichst voruurtheilsfrei untersuchen. Da wir die uxedu an den Genitalien und in der Leiste bereits constatirt haben, und der yor uns stehende Kranke vorläufig nur die Beckengegenden entblösst hält, so sehen wir uns nach ambular. torischer Manier sogleich den After an, und richtig finden sich daselbst unter einem Heer von Krankheiten auch:

Taf. 31. "Ein anderes (Recept) uzedu am Anus zu vertreiben: Feigen, Seessalz, Weihrauch, Horn ron der Kuh, zu einer Kugel formen und in den Anus bringen." 
Taf. 32. "Mittel Brennen am Anus zu verhindern, wenn er leidet in Verbindung mit uqedu in seinen (des Patienten) Beinen." - Bemerkenswerth ist, dass diesem nicht topisch, sondern durch einen Trank vorgebeugt wird.

Wir lïften nun instinctmässig die Kleider des Kranken um einen Ueberblick auf seine Haut $z u$ erlangen; auch da zeigt sich:

Taf. 29. „Ein anderes (Recept) Hautflecke zu vertreiben, die ron uzedu entstehen: Feigen $1 / 8$, Weizenbrod $1 / 32$, Sebesten $1 / 8$, grüne Bleierde (?) $1 / 32$, Wasser $1 / 3$, feucht stehen lassen, 4 Tage einnehmen. “"

"Andere Mittel Hautflecke zu vertreiben, die von nyedu entstanden sind : šeneft ${ }^{35}$ )-Samen, Zwiebeln (?), grïne IBleierde (?), šaša ${ }^{36}$-Samen, Harz von Icanthıs, Harz von T'erpenthin, Kuhmilch (gleiche Theile), gut durchkuchen und 4 Tage trinken."

Aber nicht bloss imnerlich worden die nqedu der Haut behandelt :

Taf. :30. „Ein ankleres uzedu zu tilgen: Weizenmehl, Inuramehl, Wehl von der Dumpalmenfrucht, yet ${ }^{37}$ )-Pflanze, Honig (gleiche 'Theike), als I'flaster" anfleg(n."

"Win anderes citernde Wunden zu heilen, die ans uyedn 'ntstehen: Gequetschte Dumpalnunfrucht, siisses Bier, Bolnen, Ilar\% von Icanthus, Charpir von loinwand, siisse Myrthen, sïsses Bier zusetzen (gleiche Theike), darauf als I'flaster legen." llarauf folgt eine lange Beschwörungsformel, diese Medicamente wirksamel' zu machen.

Dieser Befund zwingt uns runächst den Zungenspatcl in die Hand, um hach dem Mund zu sehen:

Taf. 27. "lin anderes u\%edu im Mund zu vertreiben." lis folgen drei verschieden lange Compositionen, durch welche u\%edu im Munde örtlich and innerlich behandolt wird.

lin sorgloser oder wenig erfahrener Arzt, welcher sich bei so Horider uyedu nicht bei Zeiten um die Augen seines Kranken kiimmert; und da boginnt dem schon

Taf. 55. "Dis Buch von den Augen. Zu machen gegen die zunalume der nzedu im Blut in dem Auge."

Taf. 56. "Behandlung der wachsenden uedu (in dom nug( ). Larauf folgt unmittelhar: 
"Ein anderes zu machen gegen Verschleierung im Auge den ersten Tag: Wasser rom Vogelteich; den zweiten Tag: Honig, Collyrium (gleiche Theile), auf einen Tag. "Hirschberg. ${ }^{38}$ ) hält diese Verschleierung für entzündliche Hornhauttrübung, Ebers für iritische Affection.

"Ein anderes zum Fernhalten der uxedu in den Augen: Collyrium 1, Dinte 1 die Augen damit salben."

Taf. 57. „Ein anderes für das Zusammenziehen der Pupille des Auges: Feilspäne von Ebenholz 1, sa ${ }^{39}$ )-Samen von Oberägypten 1, in Wasser mischen und oftmals auf die Augen thun."

Taf. 60. ". . Grinspan ... er vertreibt ihm Wasser, Eiter, Blut, Augenentzündung, Eiterfluss, Blindheit, Triefauge, die bewirkt der Gott des Fiebers, aller Todesarten, der uyedu jeder Art und aller bösen Dinge in diesen Augen, soviel ihrer sind."

"Ein anderes von der Augensalbe uxedu in den Augen abzuwehren: Spitze der Papyruspflanze, Zwiebeln (?), Honig, Gänseschmalz zu gleichen Theilen; hernach mit Vorsicht auf die Augen thun, unzählige Male."

Auch über die uxedu in den Knochen finden sich mehr weniger deutliche Hinweisungen; mitteninne der uzedu der Haut steht:

Taf. 30. "Andere Mittel für die Vorderseite des Schienbeins: Das Innere des nār-Fisches, ${ }^{40}$ ) das innen in seinem Kopf sich findet, in Honig weichen, als Pflaster auflegen, um den Kranken sogleich zu heilen." Das Wort ,sogleich" kann nicht abhalten an uжedu der Tibia zu denken, denn derselbe Ausdruck wiederholt sich ziemlich oft bei den schwersten Erkrankungen dieser und anderer Art. Hierhergebörig mag auch sein :

Taf. 107-108. "Lehre vom Tumor der uxedu. Wenn Du einen Tumor der uzedu im Kopf triffst.... und findest, dass er Flüssigkeit producirt, dass er unter Deinen Fingern, die ruhig gehalten werden, gewachsen ist, und dass er erweicht ist, wenn $\mathrm{er}^{*}$ auch nicht gross ist, so sag Du dazu: es ist ein Tumor der uzedu im Kopf . . . . Ich werde die Krankheit behandeln. Behandle sie mit dem Messer, aber pass auf, dass Du das Gefäss meidest. Es fliesst etwas daraus wie Kuchenwasser; es ist eine zottige Hülle darauf (festgebunden); lass nicht etwas davon zurück, 
lass es nicht herumlaufen; heile es so wie man eine offene Wunde heilt in jedem Körpertheil einer Person durch Umhüllen und Heilen der metu; ${ }^{11}$ ) die Flecken, die eine Person bekommt, lassen es aufschwellen und vertreiben es nachher."

So einfach es bisher war nach dem eigenen eingeschlagenen Gedankengang den alten Aegyptern nachzugehen und die oft erwähnte Krankheit zu deuten, ohne dass es nothwendig gewesen wäre dem Verfasser des Papyrus E bers mehr zu zumuthen, als man jedem alten Culturvolke, welchem alle anatomischen Kenntnisse abgehen, zumuthen kann, gesunde Sinne nämlich, so gestaltet sich jedoch die weitere Betrachtung der u\%edu darum scheinbar schwieriger, weil Alles was nuu folgt auch nicht einmal annähernd, weder in der medicinischen Literatur des Alterthums noch in der des Mittelalters, angedeutet ist; erst im Beginn der Neuzert finden sich darüber bei einigen Schriftstellem etliche blasse Spuren, die zwar schon von Paracelsus ${ }^{42}$ ) zu deutlichen Vorstellungen geformt, dennoch erst in deu letzten Decemien unseres Jahrhunderts als unerschiitterliche Thatsachen festgestellt werden konnten. Die Schwierigkeit noch weiter zu gehen, ist jedoch, wie schon gesagt, nur eine scheinbare, denn wir brauchen noch immer nicht an meln als an die gesunden Simue der alten $\Lambda$ egypter zu appelliren, um zu begreif('n, warum sie einer chronischen, constitutionellen lirkrankung, welche die uqedu offenbar war, auch noch andere Localisationen und lirscheinungen zugeschrieben haben, als die bereits rorgeführten; $j a$, es entspricht doch der niederen Bildungsstufe eines Beobachters jedenfalls weit besser, einer einmal constatirten oder anamnestisch erhobenen Krankbeit kritiklos alle diejenigen Lrscheinungen beizuzählen, welche in ihrer unmittelbaren oder mittelbaren Folge auftreten, als erst lange zu untersuchen, ob nicht etwa zwei oder mehr verschiedene Krankheitsindividuen neben- oder nacheinander verlaufen. Dies erwogen wird uns die uzedu, wo sie auch immer noch vorkommen mag, nicht mehr iiberraschen, und wir werden auch in den weiteren Ausfiihrungen durchaus uicht den Scharfsinn der alten Aegypter, sondern die Launen des Zufalls, welcher hierin das Richtige traf, bewundern können.

Zunächst begegnen wir der Krankheit beroits im ersten 
Abschnitte des Papyrus Ebers unter den "Krankheiten des Leibes"..33) Das erste Recept spricht allerdings nur dafür, dass uxedu auch durch Abführmittel behandelt wurde:

Taf. 11. Fin anderes den Leib zu entleeren und uyedu zu tilgen: Eine Prise hemit, zermablen, zerreiben, in 4 Kuchen backen, in Honig aufweichen und von der Person zu essen." "Win anderes: Grïnspan $1 / 64$, Honig ebensoviel."

Taf. 23, "Mittel uqedu im Leib zu heilen: Fleisch von einer lebenden Kuh $1 / 3$, Weihrauch $1 / 64$, Lactuca $1 / 8$, Beere rom uān-Baum $1 / 16$, frischgebackenes Brod $1 / 8$, süsses Bier $1 / 3$, durchseihen und 4 Tage einnehmen."

„Ein anderes uzedu im Leib fortzubringen: 9am-Pflanze $1 / s$, Sebesten $1 / s$, sezept-Saft $1 / 3$, süsses Bier $1 / 2$, durchseihen, kochen, 4 Tage einnehmen."

Dieselbe Tafel enthält noch zwei andere Recepte gegen uzedu „im Leibe"; Taf. 24 hat deren vier gegen dasselbe, woron eines zugleich auch für die "tödtliche āan-Krankheit" gebraucht wird; Taf. 25 hat zwei für uzedu „im Leibe" wovon eines auch für die uha-Krankheit. Diese vielen Recepte zeigen uns eben nur, dass die uxedu ziemlich oft in den Organen der Bauchhöhle entweder bloss vermuthet wurde oder auch vorgekommen ist.

Taf. 35-36. „Ein anderes Brennen der uxedu am Rumpf zu vertreiben: Feigen, Beere vom Weinstock, Sebesten, Beere vom uān-Baum, Weihrauch, Nasturtium, Kümmel, utā ron Datteln, süsses Bier (gleiche Theile), kochen, durchseihen, und 4 Tage einnehmen."

"Fin anderes uzedu am Rumpf abzuwehren: Harz von Acanthus $1 / 8$, suisses Bier $1 / 3$, zerreiben, feucht stehen lassen; durchseihen und 4 Tage trinken."

Das Vorhergehende wird jedenfalls als uyedu in der Musculatur und den Knochen des Rumpfes zu deuten sein; ob im Folgenden an das Rückenmark gedacht werden kann, mag dahingestellt bleiben:

Taf. 40. „Wenn Du eine Person untersuchst, die das Leiden an ihrem ro-ab hat und findest es an ihrem Rückgrat wie die Beschwerden von Messerträgerkrankheit, ${ }^{\mathbf{4}}$ ) so sag Du dazu: das sind die uxedu, die in seinen Rücken übergegangen sind; er ist 
krank, ich will die Rücken-Mittel machen ... Mach Du ihm eine Topfhefe und mach ihm nachher folgende Mittel: zetPflanze (frutex hortensis), Pfefferminz, Harz von Acanthus, Maurerlehm, zermahlen, kochen in Hefe von süssem Bier und 4 Tage aufstreichen, um ihn sogleich zu heilen."

Wie die alten Aegypter dazu kamen Herzkrankheiten zu diagnosticiren, ist vorläufig wohl anerfindlich, aber sie sprechen davon:

Taf. 45. "Lin anderes das Herz zu heilen und die uzedu fernzuhalten (eigentlich: fortgehen zu lassen)."

Ausfïhrlicher, wenn auch nicht deutlicher, ist über dasselbe:

Taf. 101. "Wenn das Herz sich verzehrt, so ist es eine Ansammlung von Blut im Her\%en. Wenn es die mas-Krankheit des Herzens durch uzedu ist, so ist sein Herz klein im Innern seines Bauches; die uzedu fallen auf sein Herz; er ist áat-krank, er ist mas-krank. Wenn es die Schıäche (Bitterkeit) in Folge von Alter ist, so sind es uxedu an seinem Herzen."

Besonders beweisend für den gefahrlichen und constitutionellev Charakter unserer Krankheit sind:

Taf. 46. „Der Beginn von den Mitteln, welche der Gott rä für sich selbst gemacht hat.... um die göttlichen und alle Arten tödtlicher Lirankheiten und die azedu jeglicher Art in jeglichem Glied einer Person zu vertreiben, um sie sogleich gesund zu machen."

Taf. 47. Das fünfte Mittel von der Göttin nut für den Gott ra selbst gemacht . . . und damit bestreichen alle Wunden von uyedu jeglicher Art und von allerlei Uebeln."

Taf. 104. "Lehre vom Gewächs, ${ }^{45}$ ) das in Verbindung mit Belästigung der u₹edu in jedem beliebigen Körpertheil einer Person entsteht. Wenn Du ein Gewächs triffst, das in Verbindung mit Belästigung der uqedu entstanden ist, Du findest es wie Bohnen, wie kranke Beulen ${ }^{46}$ ) entstehen auf seiner Haut, freilich nicht gross; wenn der Patient krank ist an (Wund) eiter innen in seinen Körper, so sag Du dazu: er hat ein Gewächs der uzedu, das (Wund) eiter entwickelt. Ich werde die Krankheit behandeln. Mach Du dagegen die Mittel, die von Pusteln befreien und Kiter ausziehen." 
Als "ganz unsicher in der Uebersetzung". bezeichnet Joachim:

Taf. 85. „Ein anderes Paroxismen der uzedu im zitternden Arm zu vertreiben." $\mathrm{Zu}$ bemerken wäre, dass diesem unmittelbar die "Krankheit der Zunge" folgt, wobei allerdings ron uxedu nicht die Rede ist. Unverständlich, solange wir nicht durch neue Funde nähere Aufschlüsse über die Pathologie der alten Aegypter erhalten, ist:

Taf. 40. "Wenn Du eine Person mit Verhärtung an ihrem ro-ab untersuchst; sie bricht und ist sehr krank, ihr Leiden ist wie eine Wunde, so sag Du: gehoben sind die uqedu, sie umschlingen ihn nicht (eigentlich: sie legen keinen Strick um ihn)."

Taf. 89. "Das Wachsen der uxedu in den Zäbnen zu vertreiben." Wenigstens ist nicht bekannt, dass die alten Aegypter Mercur oder andere auf die Zähne und das Zahnfleisch einwirkende Mittel angewendet haben, und sonach in den viel später und häufig vorgekommenen Irrthum verfallen konnten, die Wirkungen des Arzneimittels für Erscheinungen der Krankheit zu halten.

Taf. 38. "Untersuchst $\mathrm{Du}$ eine Person mit Verhärtung ihres ro-ab, so leg Deine Hand darauf. Findest IDu, dass ihre zait sich verstärkt hat $z$ wischen den darauf gelegten Fingern, so sag Du ihr: es ist die se en $^{47}$ )-Krankheit der uyedu, die nicht gehoben ist."

Eine letzte Reihe ron Stellen im Papyrus Ebers bezieht sich theils auf die allgemeine Therapie der uxedu, theils lässt sie auf Complicationen mit anderen Krankheiten schliessen:

Taf. 26. "Mittel uha-Krankheit zu vertreiben und uyedu zu trennen." "Ein anderes uha-Krankheit zu vertreiben und uxedu zu entfernen."

Taf. 27. „Ein anderes uxedu zu vertreiben: Mohnpflanze, Mennige, grüne Bleierde (?), Honig, sesqa-Körner, Frucht der Dumpalme, Kuchen, senen-Myrrhe, aber ${ }^{48}$ )-Oel, Fett, in Eins machen und dagegen geben."

Taf. 31. "Ein anderes āaā-Krankheit bei einem Patienten zu vertreiben, uzedu zu heben, heftige Schmerzen (Kolik), die über eine Person kommen, zu vertreiben, und den Anus durch Kïhlen zu heilen: Absynth 1/s, Beere rom uān-Baum 1/16\% 
Honig $1 / 32$, süsses Bier $1 / 2$, durchseihen und 4 Tage trinken." Taf. 45. "Handmittel ${ }^{49}$ ) den Tod im Leib zu vertreiben, zu vertreiben die göttliche, tödtliche ăā-Krankheit und die uxedu, und alles, das krank ist, zu tilgen."

Taf.' 48. „Ein anderes für seinen kranken Kopf und uxedu zu vertreiben."

Dass unter der uxedu weder ein Schmerz, noch eine Anschwellung, noch eine schmerzhafte Anschwellung, noch sonst irgend ein einzelnes Symptom, sondern eine schwere, chronische und constitutionelle Krankheit sui generis $\mathrm{zu}$ verstehen ist, ergibt sich dem Arzt nicht nur aus der aufmerksamen Lectüre des aus dem Papyrus Ebers hier Vorgeführten, und noch besser aus dem Zusammonhang desselben, sondern vicl bequemer aus dem grösseren medicinischen Papyrus des Berliner Museums, ${ }^{50}$ ) in welchem die u\% edu folgendermassen geschildert werden: ,Sein Unterleib ist schwer, der Mund seines Magens ist krank, sein Herz brenut, seine Kleider hängen schlaff herab, selbst viele Kleider vermögen ihn nicht zu erwärmen; in der Nacht quält ihn der Durst, sein Geschmack ist verdorben wie bei rinem Menschen, wolcher die Feigen der Sykomore gegessen hat, sein Fleisch ist abgestorben, wie bei einem Menschen, der sich übol befindet; geht er zu Stuhle, so versagt sein Leib die Intleerung ... In scinem Unterleibe ist lintzündung, der Geschmack scines Ilerzens ist krank; wonn er sich erbebt, so gleicht (re einem Menschen, den man hindert (sich zu bewogen)." Wenn Brugsch, der Herausreber dirses I'apyrus, democh einen '/usammenhang der uyedu mit pinguis, crassus und obesus vermuthet, so mag dics viclleicht etymologisch gegriindet sein, rom ärztlichen Standpunkt ist dies wohl nicht möglich.

Von den iibrigen im Papyrus Eibers vorkommenden Affectionen wären noch die $\bar{a} a \bar{a}-$ und die uha-Krankheit in differential-diagnostischer Richtung in Betracht zu ziehen, da sie nicht nur wirklich einige Aehnlichkeiten mit der u\%erlu erkennen lassen, sondern auch wiederholt nebeneinander angefülurt werden. Lüring ${ }^{51}$ ) vermuthet in der ăă-Krankheit die Satyriasis oder den Samenfluss und wird dazu durch das I)eterminativ (ein männliches Genitale, aus dessen horizontal gezeichnetem, also halberigirtem Penis ein wenig Flüssigkeit fast 
senkrecht abläuft) bewogen, Dieses Determinativ dürfte wohl viel eher an den Tripper erinnern; doch widerspricht sowohl dieser als auch der Annahme $L$ ï ring's ausser manchem anderen besonders der Umstand, dass die āaā-Krankheit wiederholt und ausdrücklich als eine tödtliche bezeichnet wird.

Weit grössere Aehnlichkeit mit der uxedu hat offenbar die uha-Krankheit; wir finden diese ebenfalls: „im Fleisch“, „,im Leib“, „im Körper“, „in jedem Körpertheil“ und ,jedem Gliede einer Person", in den Augen; auch sie setzt „Geschwulst mit stinkender Materie", und wird in directe Beziehung zu "Hitzblattern" und einmal auch, aber sehr lose und unsicher zu u uedu im Munde gebracht; jedoch ist ein Weiteres über die Localisation und Symptomatologie nicht verlässlich ausgedrückt, und daher selbst eine Wahrscheinlichkeits-Diagnose weniger leicht möglich, obzwar es sich, besonders bei Berücksichtigung der etymologischen Bedeutung des Krankheitsnamens ${ }^{\mathbf{5}}$ ) und der eingeleiteten Salbentherapie, an Lepra denken lässt.

Anders verhält sich dies mit der uzedu. Die Geschichte der Krankheiten hat bisher keine ihrer Familien und Individuen geschildert, deren Kennzeichen eine grössere Uebereinstimmung miteinander zeigen, als die durch blosse Stichworte angedeutete uxedu im Papyrus Ebers und die Syphilis, wie wir diese heute kennen. Wenn die Beschreibung der uqedu im grösseren Berliner medicinischen Papyrus auch kein frappantes Bild der Syphilis gibt, so widersprechen die einzelnen Züge dennoch nicht geradezu manchen Spätformen der Syphilis, besonders dann nicht, wenn man die Bezeichnung der anatomischen Regionen stets unter dem Eindrucke liest, dass die alten Aegypter noch keine exacte Terminologie besassen. Das Bild der uxedu im Papyrus Brugs ch entspricht eigentlich einem chronischen Marasmus, welcher ebenso gut durch Syphilis als durch viele andere Krankheiten veranlasst sein kann; die Diagnose: syphilitischer Marasmus, war erst durch den Papyrus Ẻbers möglich.

Die Bezeichnung uzedu oder uxetu scheint übrigens eine mythologische Abstammung zu haben, denn in dem altbabylonischen Heldengedichte "Izdubar (Nimrod)" kommt eine Hierodule der Istar (Göttin der sinnlichen Liebe etc.) namens Uchat vor, mit welcher der Freund des Helden sechs Tage und 
sieben Nächte buhlte und bald darauf von einer entsetzlichen Krankheit, jedenfalls Syphilis, befallen wurde und daran starb. Die Differenzen in Zeit und Raum zwischen der Abfassung des Papyrus Ebers und der Izdubar (Nimrod)-Sage können dieser Annahme wohl kaum entgegen sein. ${ }^{53}$ ).

Hätte auch das Wort uxedu die etymologische Bedeutung für "Schmerzen", "Geschwulst", "schmerzhafte Anschwellungen", so widerspräche dies keineswegs der angenommenen pathologischen Bedeutung, denn die Geschichte weiset auch hierin eine Reihe von Analogien nach. Unter den zahlreichen Benennungen, welche die Syphilis im Laufe der Jahrhunderte bei den rerschiedenen Volkern erhielt, finden sich auch: Venusalgie, Blattern, grosse Blattern (in den rerschiedensten Uebersetzungen), wilde Warzen, warzige Pocken, Morbus pustularum turgescentium, Buas, Bubas, Buvas, Mal de las Buas, Enfermedad de las Bubas etc.; kurz, es lässt sich wohl kaum etwas auffinden, was der Amnahme entgegen wäre:

Die u\%edu rer alten Aegypter ist unsere Syphilis.

\section{Literatur und Anmerkungen.}

1) 'Odvowairs, o, 227-232.

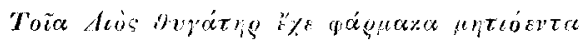

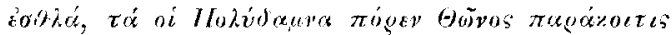

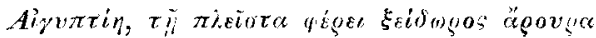

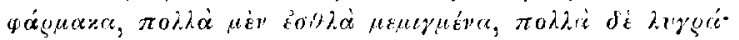

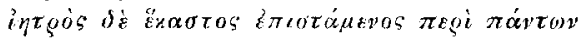

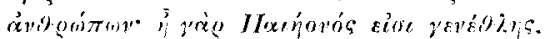

2) Horodot, Geschichten, Il., 77-84. - Vorgl. Curt Sprengel, Geschichte del Alzmoikunde. 3. Aufl. Halle, 1821, 8*, I., p. 61-102.

3) Diodor Siculns. - Vergl. eberda.

4) Clemens von Alexandria, Stromata, libr. VI., cap. IV.

5) Galenus, De facult. simplic. medicament. libr. VI. Praef. edit.

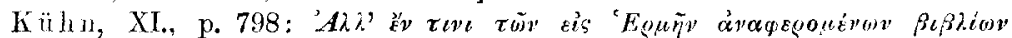

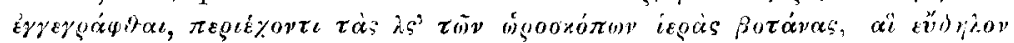

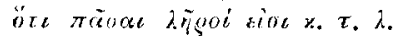

6) Eber.s. Papylus Ebers. Das hermetische Buch über die Arzncimittel der alten Acgypter in hiontischer Schrift. Teipzig, 1875, Fol. Il.

7) Hirsch, August. Geschichte der Augenhejlktunde. Sonderdruck 
aus Graefe und Saemisch, Handbuch der Augenheilkunde. Leipzig, $1877,8^{0}$, VII., p. 237.

8) Joachim, H. Papyrus Ebers. Das älteste Buch über Heilkunde. Aus dem Aegyptischen zum ersten Male vollständig übersetzt. Berlin, 1890. $8^{\circ}$. pp. XX., 214.

9) Jamblicbus. De mysteriis Aegypt. lib. VIII, cap. IV. - Vergl. Curt S prengel Geschichte der Arzneikunde. Halle 1821, $8^{\circ} \mathrm{T}$, pag. 78.

10) Das hier folgende Recept ist widersinnig und entfällt daher auch wie andere unbedeutende.

11) Brugsch übersetzt „bennut" mit Pustel; nach Joa chim ist der Stamm „rund oder kugelig fein".

12) hekenun: köstlich lieblich.

13) genti : Cassia.

14) nehedet: Zahnkraut.

15) hunta: „besondere Pflanzenart, deren Dasein mit dem Hinterland von Apollinopolis in Verbindung gesetzt wird“ $\mathrm{Brugsch}$, Wörterbuch, VI., p. 798.

16) neša : Gerste.

17) a. āt: kratzen, ritzen, zerkratzen.

18) mahui : Wunderfrucht.

19) des̃er : Name einer besonderen Art (runder) Opferbrode. Brug s c h, Wörterbuch, VI., p. 470; Joachim, H. p. 67.

20) Lüring, Heinrich L. Emil. Die über die medicinischen Kenntnisse der alten Aegypter berichtenden Papyri verglichen mit den medicinischen Schriften griechischer und römischer Autoren. Leipzig, 1888, $8^{\circ}$, p. 170 .

21) šepen: Die Räude, nach Prof. Lieblein, Aegyptologe an der Universität Christiania; Vergl. Joachim p. 155 und Vorrede p. VI.; „rothe Flecken zeigen", nach Brugsch, Wörterbuch, VII., p. 1181.

22) L üring, p. 39.

23) Lüring hat in der früheren Stelle, in welcher Joa chim ebenfalls "Körper" hat mit "Fleisch" übersetzt; es wird dies wohl auch hier stehen, und die Bedeutung Genitalien haben sollen, was doch auch die Worte "eines Mannes oder einer Frau" andeuten.

24) Für Wunde steht.im Original $\chi$ art, was nach Brugsch, Wörterbuch, VI., p. 884 „Krankheit in ihren verschiedenen Formen, Schwäche bedeutet.

25) ro-ab: Herzgrube, Herzgegend, Magengrube nach Joachim p. XVIII.; Nabel, Nabelgegend, Herzgrube nach L ür ing, p. 74.

26) zait: Krankheit.

27) Für nGeschlechtsorgane" steht "henut" des Körpers; die Uebersetzung ist nicht sicher.

28) Lüring übersetzt: Hüfte.

29) senenutet: vielleicht Pistia Stratiotes L. nach Lüring p. 161.

30) Simon, F. A. Kritische Geschichte des Ursprunges, der Patho- 
logie "und Behandlung der Syphilis, Tochter und wiederum Mutter des Aussatzes. Hamburg, 1858-60, 80, III.

31) Joachim, p. 119.

32) Haeser, Heinrich. Lehrbuch der Geschichte der Medicin. Dritte Bearbeitung. Jena, 1875, $8^{\circ}$. I., p. 53.

33) Lüring, p. 17.

34) Joachim, p. XIX.

35) šeneft: Opferkorn.

36) šaša: Kuchen aus zusarnmengepressten Rosinen.

37) yet: frutex hortensis.

38) Hirschbexg bei Joachim p. 82 .

39) sa-Samen: Natron oder Salpeter.

40) nār-Fisch: Wels.

41) meta: Adern, Gefïsse, Muskeln, Nerven, Sehnen.

42) Paracelsis, Chirurgische Bücher vnd Schriften, durch Joh. Huser. Strassburg, 1618, fol., p. 795 .

4:3) Unter "Leib" im engeren Sinne werden woht inmer die Gedärme, in weiteren Sinne alle Bauchorgane einzeln und zusammen zu verstehen sein.

44) Diese Krankheit ist nach Joachim in Berliner med. Papyrus beschrichen.

45) Gewächs: ägyptisch humhunt übersctzt $S$ tern in seinem Glossar zum Papyrus Ebers mit tumor serophulosus, struma. Vorgl. Joachim, 0. 187.

(6) Lüring, übersetzt Geschwüre.

47) seyen: Geschwür, nach Brugsch, Wörterbuch, VII. p. 1109.

18) aber-ocl: ein heiliges Salböl.

49) Handmittel: augenblicklich anzuwendende Mittel.

50) $\mathrm{H}$ a $\mathrm{escr}$, I. c. I., p. 59 .

51) Lüring, p. 21.

53) uhau (nach Joachim uha): "rorheesende", nud wie l, üring meint, vielleicht auch "verbrennende hrankhoit".

55) Prokseh, J. K. Die Syphilis bei den alten Babyloniern und Assyriein. - In: Monatsh. f. prakt. Dormat. Hamburg, 1891, XIl., p. 38.1-393. 